analytischen Funktionen heranziehen, die im Zwischengebiet zwischen dieser Kurve und dem in Abb. 3 gezeichneten Rechteck regulär sind und welche die auf den Rechteckseiten vorgeschriebene Randbedingung (s. § 2) erfüllen. Dies sind jedoch gerade die Ableitungen beliebiger Ordnung $\frac{\mathrm{d}^{n} Z}{\mathrm{~d} z_{2}{ }^{n}}$ der Funktion $Z=$ $Z\left(z_{2}\right)$ Gl. (5). Man kann also das komplexe Potential $\mathbf{Z}$ in eine Multipolreihe

$$
\mathbf{Z}=\sum_{n=0}^{\infty} c_{n} \frac{\mathrm{d}^{n} \mathrm{Z}}{\mathrm{d} z_{2}{ }^{2}}
$$

mit komplexen Koeffizienten $c_{n}$ entwickeln. Die $c_{n}$ sind so zu bestimmen, daß

$$
\mathfrak{R e} \mathbf{Z}=\Phi_{0} \quad \text { für } \quad|w|=\frac{1}{2} d
$$

ist. Bei der praktischen Durchführung wird man natürlich statt mit der unendlichen Reihe Gl. (39) mit einem Aggregat von endlich vielen Gliedern rechnen und zur Bestimmung der Konstanten aus Gl. (40) die entsprechenden höheren Potenzen von $\delta$ vernachlässigen.

Für Anregungen und Ratschläge habe ich Herrn Prof. Dr. E. F u e s, Herrn Prof. Dr. E. S c h o p p e r und Herrn cand. phys. H. Jür ge n s e n zu danken. Ich freue mich, diese Arbeit Herrn Prof. Dr. E. R e ge n e r zu seinem siebzigsten Geburtstage als einen Beitrag zum wissenschaftlichen Arbeitsprogramm seines Instituts widmen zu können.

\title{
Untersuchungen an Geiger-Müller-Zählern mit mehreren Zähldrähten
}

\author{
Von H. Jürgensen und H. M. Weiss \\ Aus dem Physikalischen Institut der Technischen Hochschule Stuttgart \\ und der Forschungsstelle für Physik der Stratosphäre in der Max-Planck-Gesellschaft Weißenau \\ (Z. Naturforschg. 6 a, 694-698 [1951]; eingegangen am 9. August 1951)
}

\section{Herrn Professor Erich Regener zum 70. Geburtstag}

Die Zähleigenschaften von Auslösezählern mit mehreren Zähldrähten zwischen ebenen bzw. konzentrisch gekrümmten Platten werden im Hinblick auf die Verwendung als Großflächenzähler untersucht. Die Entladungen sind nicht auf den Zählbereich der einzelnen Zähldrähte lokalisiert; das Übergreifen der Entladung wird untersucht. Als praktische Anwendung wird eine Antikoinzidenz-Abschirmung durch einen Mehrdraht-Kreisringzähler gegenüber einem Zentralzählrohr zur Zählung geringer Intensitäten beschrieben.

Z ur Anwendung auf spezielle Probleme wurde früher von E. R e g e n e r die Untersuchung von Zählrohren nichtzylindrischer Form angeregt ${ }^{1}$. Im folgenden berichten wir über Messungen an Auslösezählern mit mehreren Zähldrähten zwischen parallelen ${ }^{2}$ bzw. konzentrisch gekrümmten Platten ${ }^{3}$.

\section{Zähleigenschaften eines Mehrdraht- Parallelplatten-Zählers}

Der Aufbau des Zählers ist aus Abb. 1 zu ersehen: In der Mittelebene zweier paralleler Metallplatten sind mehrere äquidistante Zähldrähte gespannt. Die Anordnung entspricht einer in einem einzigen Zähler zusammengefaßten Zählrohrlage. Ein solcher Mehrdrahtzähler bietet gewisse Vorteile als Großflächenzähler oder als Antikoinzidenzabschirmung.

Der für die Zähleigenschaften maßgebende Feld-

1 E. S a u r, Diplomarbeit, Stuttgart 1934.

2 H. M. W e i $\beta$, Diplomarbeit, Stuttgart 1950. verlauf unserer Anordnung ist für parallele Platten noch einfach berechenbar. Für konzentrisch gekrümmte Platten (sog. Kreisringzähler) hat S e e g e $\mathrm{r}^{4}$ eine geschlossene Lösung angegeben. Der experimentell im elektrolytischen Trog ermittelte Feldverlauf ergab das in Abb. 1 gezeichnete Bild. Von den Kathodenplatten ausgehend, verläuft das Feld homogen bis zu einer Entfernung von der Drahtreihe, die etwa dem halben Drahtabstand entspricht. Die Entfernung der Zähldrähte darf also höchstens so groß sein, daß von einem senkrecht durch das Zwischengebiet fliegenden Teilchen im homogenen Feldbereich noch mindestens ein Ionenpaar erzeugt wird. In der untersuchten Anordnung war $d=9 \mathrm{~mm}, D=20 \mathrm{~mm}$ (Abb. 1). Bei der Abtastung des Zählers an verschiedenen Stellen mit einem schmalen $\gamma$-Strahlbündel erhält man über den gesamten Zähler hinweg bis auf die unmittelbaren Randgebiete eine konstante Impulszahl.

3 H. J ür g e n s e n, Diplomarbeit, Stuttgart 1951.

4 A. S e e g e r, Z. Naturforschg. 6a, 688 [1951]. 
Ebenso wie bei der Parallelschaltung von getrennten Zählrohren ist gleiche Einsatzspannung der einzelnen Drähte Voraussetzung für einen hinreichend großen Zählbereich der Gesamtanordnung. Dies läßt sich ohne besondere mechanische Vorsichtsmaßregeln soweit erreichen, daß in dem untersuchten Zähler die einzelnen Einsatzspannungen um etwa $\pm 1 \%$ differierten. Der Konstanzbereich der Gesamtanordnung ist naturgemäß etwas kleiner als der eines einzelnen Drahtes, da er sich nur über das Spannungsintervall erstreckt, in dem sich die Zählbereiche der Einzeldrähte überdecken. Er betrug etwa $100 \mathrm{~V}$ für den gesamten Zähler und 115-130 V für die einzelnen Zähldrähte bei einem Impulsanstieg von weniger als 10\%. Im Vergleich zu den sonst üblichen Zählrohren

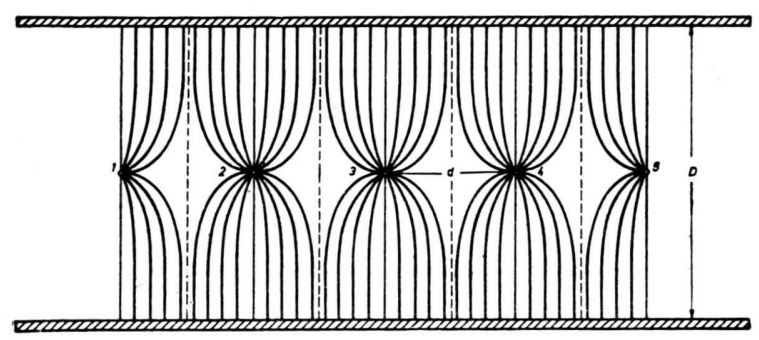

Abb. 1. Statischer Feldverlauf des Parallelplatten-Zählers.

ist bei dem Mehrdrahtzähler das Plateau kürzer, bedingt durch die ungünstigeren Feldverhältnisse in der Nähe des Zähldrahtes. Die Größe des Konstanzbereiches für die Einzeldrähte kann nämlich durch negative Potentiale auf den Nachbardrähten, welche das Feld am betrachteten Zähldraht demjenigen eines Zylinderfeldes annähern, nahezu verdoppelt werden.

Ein solcher Mehrdrahtzähler eignet sich somit als Großflächenzähler oder als Antikoinzidenzanlage gegen einen zweiten Zähler. Eine praktische Anwendung für den letzteren Fall $^{3}$ wird im zweiten $\mathrm{Ab}$ schnitt der Arbeit beschrieben. Zur Messung von Koinzidenzen zwischen den einzelnen Drähten scheint sich eine solche Anordnung hingegen nicht zu eignen; es war unmöglich, die an einem einzelnen Draht gezündete Entladung stets nur auf diesen zu lokalisieren. Mit wachsender Überspannung (= Spannung über der Einsatzspannung) greift vielmehr die Entladung in zunehmendem Maße auf die übrigen Drähte über. Die Wahrscheinlichkeit, mit der die Entladung zwischen zwei Drähten, z. B. 2 und 4 in Abb. 1, bei verschiedenen Gasfüllungen überspringt, gibt Abb. 2 wieder. Die übrigen Zähldrähte sind durch negative Potentiale von $-550 \mathrm{~V}$ von der Zählung ausgeschlossen; das Zwischenfeld um Draht 3 verhindert dabei gleichzeitig noch einen eventuellen Übergang der Entladung durch ein Ineinandergreifen der Zählgebiete der Drähte 2 und 4. Als Abszisse ist nicht die Überspannung aufgetragen, da besonders bei den Füllungen mit hohem Partialdruck des Dampfzusatzes die Einsatzspannung nur ungenau definiert war, sondern die bei einer bestimmten Verstärkung am Oszillographen auftretende Impulshöhe $A$, welche in erster Linie ein Maß für die Anzahl der in der Lawine erzeugten Elektronen ist.

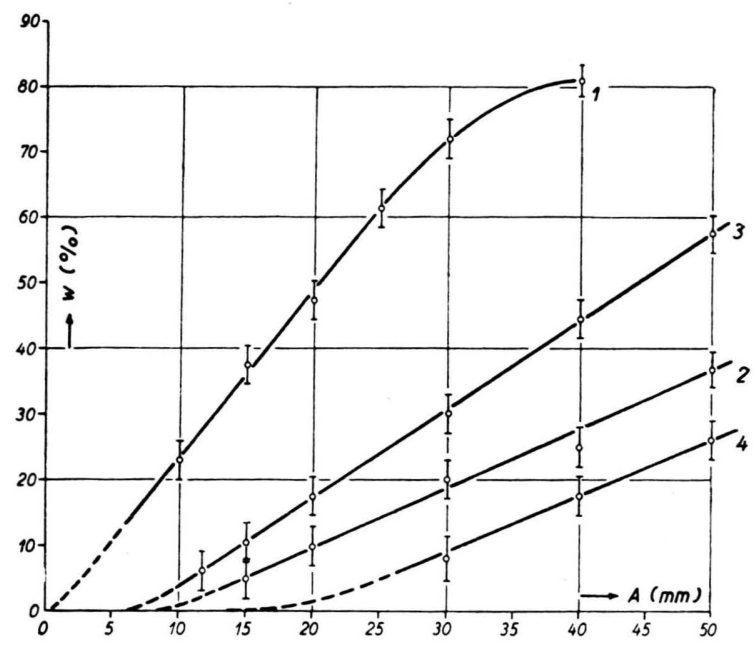

Abb. 2. Wahrscheinlichkeit für das Überspringen der Entladung auf den Nachbardraht bei verschiedenem Füllgas. Kurve 1: $15 \mathrm{~mm} \mathrm{Hg}$ Äthylalkohol + $55 \mathrm{~mm} \mathrm{Hg}$ Argon; Kurve 2: $30 \mathrm{~mm} \mathrm{Hg}$ Athylalkohol $+40 \mathrm{~mm} \mathrm{Hg}$ Argon; Kurve 3: $15 \mathrm{~mm} \mathrm{Hg} \mathrm{Methylal} \mathrm{+} 55 \mathrm{~mm} \mathrm{Hg}$ Argon; Kurve 4: $30 \mathrm{~mm} \mathrm{Hg}$ Methylal $+40 \mathrm{~mm} \mathrm{Hg}$ Argon.

In üblichen zylindrischen Zählern ${ }^{5,6}$, bei denen der Zähler durch Glastropfen auf dem Zähldraht bzw. durch Isolierstoffringe auf der Wandung in zwei Hälften unterteilt war, ließ sich die Entladung auf die jeweiligen Zählerbereiche einigermaßen lokalisieren. Im Gegensatz dazu ist bei unserer Anordnung die Lokalisierung der Entladung unschärfer. Während nämlich dort nur die in nächster Nähe der Trennstelle erzeugten Photonen einen relativ kurzen Weg in das Nachbarvolumen zurückzulegen haben, tragen bei unserem Zähler alle Stellen der parallel gespannten Drähte gleichstark zu einem Überspringen der Entladung bei, so daß die Zahl der auf kürzestem Weg in das Nachbargebiet ausgestrahlten Photonen viel größer ist und demgemäß auch die Wahrscheinlichkeit für einen Übergang der Entladung.

5 H. G. S t e ver, Physic. Rev. 61, 38 [1942].

6 M. H. Wilkening u. W. R. K a nne, Physic. Rev. 62, 534 [1942]. 
Welcher Mechanismus dem Übergreifen der Entladung zugrunde liegt, kann an Hand der vorliegenden Meßergebnisse nicht eindeutig entschieden werden. Es scheint aber kaum möglich, dafür den gleichen Effekt verantwortlich zu machen, der die Ausbreitung der Lawine längs des Zähldrahtes verursacht. Dieser beruht ja vermutlich auf einer starken Absorption der energiereichen UV-Photonen aus angeregten ArgonAtomen und -Ionen durch die Moleküle des Dampfzusatzes mit anschließender Dissoziation derselben. Von Alder und Mitarbeitern ${ }^{7}$ wurde der $\mathrm{Ab}$ sorptionskoeffizient hierfür zu $a=640 \mathrm{~cm}^{-1}$ (bei $760 \mathrm{~mm} \mathrm{Hg}$ und $20^{\circ} \mathrm{C}$ ) bestimmt. Die Schärfe der Lokalisierung der Entladung müßte dann aber bei Erhöhung des Dampfzusatzes im Füllgas weit stärker zunehmen, als unsere Messungen ergaben.

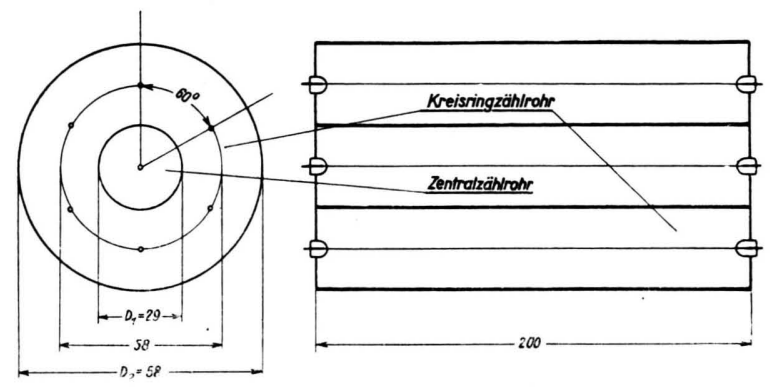

Abb. 3. Schematischer Aufbau des Kreisringzählers.

\section{Zähleigenschaften eines Mehrdraht- $\mathrm{Kre}$ isring-Zählers}

Als Kreisringzähler bezeichnen wir eine Anordnung nach Abb. 3, die aus zwei konzentrischen Zylindern als Kathoden und einer Anzahl von Zähldrähten besteht.

Er gestattet Koinzidenz- oder Antikoinzidenzmessungen in Verbindung mit einem im Innern angebrachten Zentralzählrohr über den ganzen Vollraum, mit Ausnahme des durch die Stirnflächen gebildeten kleinen Raumwinkels. Eine solche Anordnung in Antikoinzidenzschaltung ist beispielsweise zweckmäßig bei der Messung geringer Aktivitäten mit dem Zentralzählrohr unter Abschirmung der Umgebungsstrahlung durch den äußeren Kreisringzähler. Die Abschirmung kann auch angebracht sein z. B. bei der Zählung geringer Neutronenintensitäten mit einem im Proportionalbereich arbeitenden $\mathrm{BF}_{3}$-Zählrohr so großen Volumens, daß durch die Umgebungsstrahlung vergleichbare Impulsgrößen erzeugt werden können.

7 F.Alder, E. Baldinger, P.Huber u. F. Metzger, Helv. physica Acta 20, 73 [1947].
Schließlich läßt sich mit einem Kreisringzähler als $\mathrm{BF}_{3}-\mathrm{Zähler}$ die Ansprechwahrscheinlichkeit für Neutronen erhöhen, wenn außer dem üblichen äußeren Paraffin-Bremsmantel ein zweiter an Stelle des Zentralzählrohres im Innern des Kreisringes angebracht wird.

Wir haben den statischen Potentialverlauf des Kreisringzählers wiederum im elektrolytischen Trog bestimmt (Abb. 4). Die gemessenen Potentiale sind in der Zeichnung auf ein Drahtpotential $=1000 \mathrm{~V}$ umgerechnet. In der Nähe der Zähldrähte verlaufen die Äquipotentialflächen als konzentrische Zylinderflächen

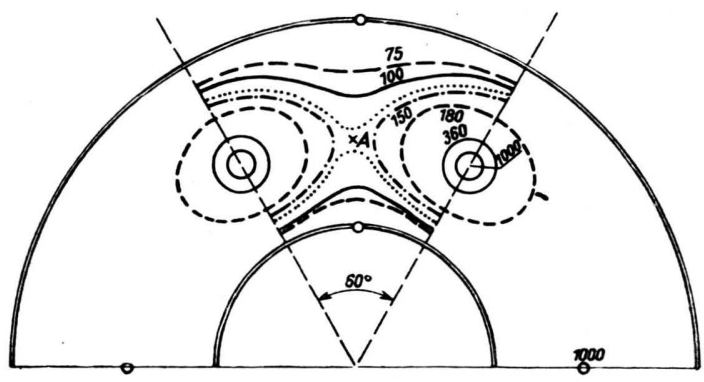

Abb. 4. Statischer Potentialverlauf im Kreisringzähler.

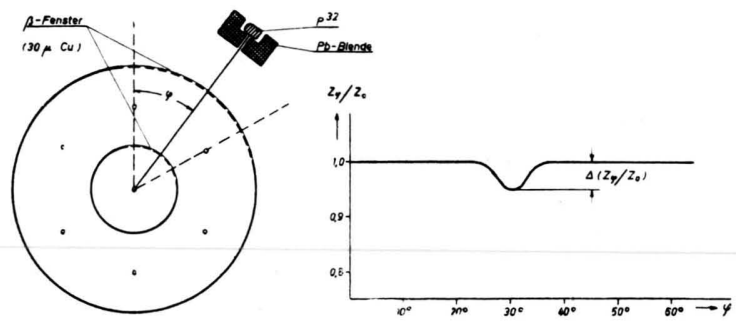

Abb. 5. Abhängigkeit der Ansprechwahrscheinlichkeit vom Einfalls-Azimut $\varphi$ der Strahlung.

um die Drähte. Das Feld entspricht in erster Näherung einem Zylinderfeld mit den Radien $r_{\mathrm{i}}=r_{\mathrm{D}}$ und $r_{\mathrm{a}}=\frac{r_{2}-r_{1}}{2}$ (Abb. 3).

In der Nähe der Zählrohrwände verlaufen die Potentialflächen als konzentrische Zylinder um die Mittelachse des Gesamtzählrohres.

Der Feldverlauf im Bereich um den Zähldraht bis etwa zur gezeichneten Äquipotentialfläche $150 \mathrm{~V}$ entspricht dem eines Zylinderzählrohres. Längs einer bestimmten achsenparallelen Linie zwischen zwei Drähten wird die Feldstärke Null (Punkt A in Abb. 4). Dieser Fall tritt im Zylinderzählrohr nicht auf.

Bei radialer Einstrahlung eines ausgeblendeten Bündels von $\gamma$-Strahlen ist eine Richtungsabhängigkeit nicht festzustellen, dagegen ist beim Beschuß mit 
schnellen Elektronen eine leichte Abhängigkeit vom Einfallsazimut $\varphi$ vorhanden. Die schematische Versuchsanordnung und das Ergebnis zeigt Abb. 5. Danach ist in der Mitte zwischen zwei Drähten die Ansprechwahrscheinlichkeit um einige Prozent verringert infolge der geringen Feldstärke um Punkt A (Abb. 4), was sich auch bei axialer Strahlrichtung zeigen muß. Ihr Anteil, bezogen auf das gesamte Zählvolumen, ist jedoch vernachlässigbar.

Da sich alle Zähldrähte im gleichen Gasvolumen befinden, müßten sie bei gleichem Potential aus Symmetriegründen die gleiche Einsatzspannung besitzen. Die Zählspannungen der Einzeldrähte differierten gegeneinander um maximal $20 \mathrm{~V}$ bei einer mittleren Einsatzspannung von $1200 \mathrm{~V}$ für den Auslösebereich. Das Zählplateau beträgt für einen gemeinsamen $\mathrm{Ab}$ -

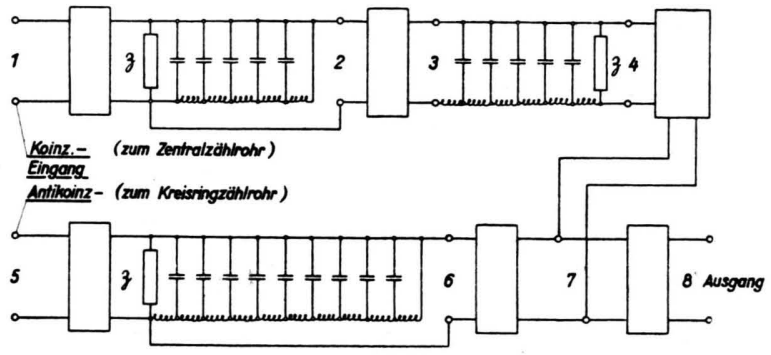

Abb. 6. Koinzidenz-Antikoinzidenz-Verstärkung.

leitwiderstand von $2 \mathrm{M} \Omega$ für alle Drähte etwa $150 \mathrm{~V}$; wird nur jeder zweite Draht an die Hochspannung angeschlossen, ist der Konstanzbereich des Einzeldrahtes $180 \mathrm{~V}$.

Beim Betrieb aller Drähte an einem gemeinsamen Ableitwiderstand im Auslösebereich treten Impulse bis zu maximal 3-facher Größe gegenüber den Impulsen am Einzeldraht auf. Die Erscheinung hat W eiß² eingehender untersucht. Sie ist darauf zurückzuführen, daß die Entladung auf die Nachbardrähte überspringt. Bei den großen Impulsen beobachtet man eine wesentlich längere Impulsdauer und eine abgeflachte Impulsspitze. Die Höhe der verschiedenen Impulse ist nicht ein ganzes Vielfaches des Grundimpulses. Die Zahl der vergrößerten Impulse steigt mit der Zählspannung und mit fallendem Druck bei gleicher prozentischer Zusammensetzung des Füllgases. Dieses Übergreifen der Entladung begrenzt, analog wie beim ebenen Plattenzähler, hier die $\mathrm{Zu}-$ ordnung des Einfallsazimuts der Strahlung zu dem jeweils ansprechenden Zähldraht.

In der Verwendung als Antikoinzidenz-Aggregat gegen ein Zentralzählrohr haben wir für die elek- trische Kopplung der Koinzidenz- und der Antikoinzidenz-Impulse eine Anordnung verwendet, deren zeitliches Auflösungsvermögen, unterhalb desjenigen der Zählrohre, $<5 \mu$ sec ist. Nach bekanntem Prinzip $^{8}$ werden Koinzidenz- und Antikoinzidenz-Impuls in getrennten Kanälen durch Verzögerungsglieder mit Reflexion (Abb. 6) in scharfe Rechteckimpulse jeweils einheitlicher Dauer umgeformt. Der Koinzidenz-

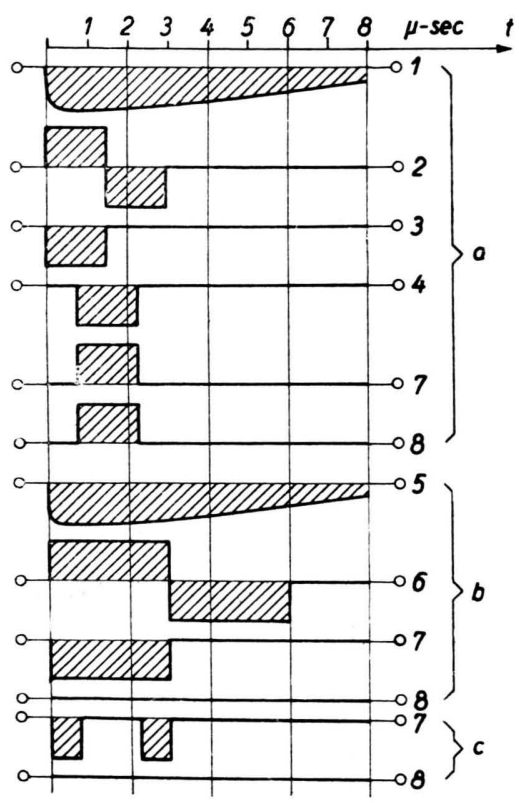

Abb. 7. Impuls-Zeitplan des Koinzidenz- und Antikoinzidenzkanals. Fall a) Impuls allein im Zentralzählrohr (Koinzidenzimpuls); Fall b) Impuls allein im Kreisringzählrohr (Antikoinzidenzimpuls); Fall c) gleichzeitiger Impuls in beiden Zählrohren.

Fall a) Impuls allein im Zentralzählrohr (KoinzidenzImpuls);

Fall b) Impuls allein im Kreisringzählrohr (Antikoinzidenzimpuls);

Fall c) gleichzeitiger Impuls in beiden Zählrohren.

impuls wird durch ein weiteres Verzögerungsglied zeitlich so verschoben, daß bei einer Mischung beider Impulse eine einwandfreie Tilgung des Koinzidenzimpulses erreicht wird. Abb. 7 zeigt den Zeitplan für die drei möglichen Fälle:

Die Wirksamkeit der Abschirmung für $\beta$-StrahlAntikoinzidenzen ist folgende: Über den ganzen Winkelbereich zwischen zwei Drähten und beim Betrieb des Zentralrohres im Auslösebereich werden Abschirmfaktoren von $98 \%$ gefunden; bei ausgeblende-

8 S. C. C u r r a n and J. D. C r a g g s, Counting Tubes, London 1949, p. 135. 
ter Strahlung nach Abb. 5 von $96 \%$ bzw. $99,8 \%$ bei einer Gesamtintensität von 900 bzw. 120 Teilchen/min.

Im Proportionalbereich und im bedingten Proportionalbereich des Zentralzählrohres mit $\mathrm{BF}_{3}$-Füllung beträgt der Nulleffekt 0,05 Teilchen/min mit Antikoinzidenzabschirmung. Er stieg bis auf 1 Teilchen/min im bedingten Proportionalbereich ohne Abschirmung.
Es lassen sich mit dieser Anordnung noch sehr geringe Neutronenintensitäten registrieren.

Dem Leiter des Instituts, Herrn Prof. Dr. E. Re gen er, danken wir für die Möglichkeit zur Durchführung der Arbeiten und für sein stetes Interesse an ihrem Fortgang. Ferner möchten wir für die Anregung und ständige Unterstützung Herrn Prof. Dr. E. S c h o p p e r danken.

\title{
Über die Fluoreszenz von Gasen bei Anregung durch $\iota$-Teilchen
}

\author{
Von A. E. GrüN und E. SCHOPPER \\ Aus dem Physikalischen Institut der Technischen Hochschule Stuttgart \\ (Z. Naturforschg. 6 a, 698-700 [1951]; eingegangen am 9. August 1951)
}

\section{Herrn Professor Erich Regener zum 70. Geburtstag}

\begin{abstract}
Das von einzelnen $\alpha$-Teilchen in Gasen angeregte Fluoreszenzleuchten wird mit Hilfe von Sekundärelektronen-Vervielfachern untersucht. Unter den „technisch reinen“ Gasen lieferte $\mathrm{N}_{2}$ und $\mathrm{Ar}$ rund 100-mal größęre Quantenausbeuten als $\mathrm{H}_{2}, \mathrm{O}_{2}$ und $\mathrm{CO}_{2}$. Zusätze von organischen Dämpfen wirken löschend. Es wird in $\mathrm{N}_{2}$ eine Quantenausbeute von 1000 Quanten im Spektralbereich von 3000 bis $4000 \AA$ je $\alpha$-Teilchen von $4 \mathrm{~cm}$ Reichweite bei Normaldruck abgeschätzt.
\end{abstract}

E $s$ ist seit langem bekannt, daß starke Präparate von $\alpha$-Strahlern das umgebende Gas, insbesondere den Stickstoff, zu sichtbarem Leuchten anregen ${ }^{1}$. Im Vergleich zu den Lumineszenz-Effekten der $\alpha$-Strahlen in festen Körpern, die auch einzelne Teilchen durch ihre Szintillation noch visuell erkennen lassen und von E. R e g e ner vor rund 40 Jahren $^{2}$ zu einer Präzisionszählung von $\alpha$-Teilchen verwendet wurden, sind die Leuchteffekte in Gasen sehr schwach und bislang nur integral untersucht worden.

Wir haben versucht, die durch ein einzelnes $\alpha$-Teilchen in Gas von Normaldruck erzeugte Szintillation mit Hilfe von Sekundärelektronen-Vervielfachern nachzuweisen. Das mit Elektronenvervielfachern erreichbare hohe zeitliche Auflösungsvermögen ${ }^{3}$ läßt erwarten, daß Einblicke in den zeitlichen Mechanismus des durch das Teilchen verursachten Anregungsund Rekombinationsleuchtens möglich sind. Ein praktisches Ziel ist der Nachweis von Teilchen durch Gasszintillation, u. a. beispielsweise zur Steuerung einer Wilsonkammer auf Prozesse, bei denen der übliche Nachweis durch die Ionen selbst nicht erfolgen soll.

Im folgenden berichten wir über einige Ergebnisse mit einer einfachen Versuchsanordnung, deren schematischer Aufbau aus Abb. 1 hervorgeht.

1 Zusammenfass. Bericht bei R. Pohl, Jb. Radioaktivität 4, 100 [1907].

2 E. R e g e n e r, Berl. Ber. 1909, 948.
Das ausgeblendete $\alpha$-Strahlbündel tritt in einen Hohlraum ein, dessen Wände von zwei Hohlspiegeln mit Aluminiumbelag gebildet werden. Eine Lichtleitung aus Plexiglas verbindet die Meßkammer mit dem gekühlten Vervielfacher (1 P 28). Der optische Wirkungsgrad dieses Systems ist in der Umgebung der Rotationsachse $10 \%$, außerhalb sinkt er schnell ab. Derselbe Hohlraum mit einem diffus reflektierenden Wandbelag aus Magnesiumoxyd hat einen Wirkungsgrad von nur $3 \%$, aber gleichmäßig im ganzen Raum (Prinzip der Ulbrichtschen Kugel). Gemessen wurde mit der ersten Anordnung; durch genügend enge Ausblendung des $\alpha$-Strahlenbündels wurde dafür gesorgt, daß der optische Wirkungsgrad innerhalb des leuchtenden Volumens nicht zu sehr schwankte.

Mit „technisch reinen“ Gasen haben wir folgende Beobachtung gemacht: Die größte Lichtausbeute (Zahl der Quanten je $\alpha$-Teilchen) wurde in Argon und Stickstoff gemessen; das Argon enthielt 2\% Stickstoff als Verunreinigung. In Wasserstoff, Sauerstoff und Kohlendioxyd war die Ausbeute mindestens 100-mal kleiner. Genauer untersucht wurde die Lichtausbeute in $\operatorname{Ar}$ und $\mathrm{N}_{2}$. Sie zeigte sich unabhängig von einem angelegten elektrischen Feld. Es handelt sich also um das Anregungs- und nicht um das Rekombinationsleuchten. Der Zusatz organischer Dämpfe, wie Alkohol, Benzol, Xylol sowie von $\mathrm{O}_{2}$, wirkt löschend. Mit Hilfe von Filtern wurde festgestellt, daß das emit-

3 K. P. M e yer, E. B a lding er u. P. H uber, Helv. physica Acta 23, 121 [1950]; N. S c h a e t t i, Helv. physica Acta 23, 108 [1950]. 